\title{
User clustering and resource allocation in downlink CoMP with NOMA
}

\author{
Norshidah Katiran', Shaharil Mohd Shah'2, Noorsaliza Abdullah', \\ Aimi Syamimi Abdul Ghaffar ${ }^{4}$, Faiz Asraf Saparudin ${ }^{5}$ \\ ${ }^{1,2,3}$ Faculty of Electrical and Electronic Engineering, University Tun Hussein Onn Malaysia, Malaysia \\ ${ }^{4,5}$ Faculty of Engineering Technology, Universiti Tun Hussein Onn Malaysia, Malaysia
}

\section{Article Info}

Article history:

Received Dec 25, 2018

Revised Mar 8, 2019

Accepted Mar 29, 2019

\section{Keywords:}

JP-NOMA-CoMP

Resource allocation

SIC

Sum rate

User clustering

\begin{abstract}
In coordinated multipoint (CoMP) system, the cell-edge performance is improved by minimizing inter-cell interference (ICI) through coordination of resources. Additionally, the non-orthogonal multiple access (NOMA) has been introduced as a promising candidate to further enhance the throughput of next generation wireless communication systems. NOMA allows multiple users to access the wireless channel in the same bandwidth simultaneously, however at different transmit power. In joint transmission NOMA in CoMP (JT-NOMA-CoMP), multiple cells jointly transmit data to users using the same time-frequency resources, which significantly improves the system performance. In this paper, we present a low-complexity user clustering and resource allocation strategy in downlink JP-NOMA-CoMP system with multiple antenna. Based on computer simulation, we show that the proposed approach outperforms the conventional JP-OMA-CoMP and single antenna JP-NOMA-CoMP in terms of achievable sum rate.
\end{abstract}

Copyright $(0) 2019$ Institute of Advanced Engineering and Science. All rights reserved.

Corresponding Author:

Norshidah Katiran,

Faculty of Electrical and Electronic Engineering,

University Tun Hussein Onn Malaysia,

86400 Parit Raja, Batu Pahat, Johor, Malaysia.

Email: norshida@uthm.edu.my

\section{INTRODUCTION}

Coordinated Multipoint (CoMP) transmission and reception is considered as one of the principle empowering technologies for LTE-Advanced. The system spectral efficiency enhancement is attained through base station cooperation where resources are allocated among users in a coordinated way [1]. For downlink, a channel allocated to a cell edge user cannot be used for other users' transmission simultaneously. Therefore, if the orthogonal multiple access (OMA) is adopted, the spectral efficiency of the system deteriorates with the increasing number of cell edge users. To overcome this issue, non-orthogonal multiple access (NOMA) can be employed [2,3].

Recently, NOMA has gained great interest from both academia and industry due to its potential to improve the spectral efficiency of transmission significantly [2], [4-6]. The key idea of NOMA is to perform simultaneous transmission to multiple users over the same resources (i.e., time, channel, space) at the expense of inter-user interference [7]. Under power-domain NOMA, a base station schedules multiple users to use the same transmission resources by superposing their signals in the power domain. The superposition is accomplished in such a way that each NOMA user can successfully decode the desired signal by applying successive interference cancellation (SIC) at the intended receiver [7].

Over the recent years, application of NOMA in CoMP systems has drawn a significant attention of the researchers. ICI is the major issue in multi-cell NOMA systems, as it reduces a cell-edge user's performance. The fundamentals of the combination of NOMA and CoMP in downlink transmission was 
investigated in $[5,7]$. Several up-to-date interference management techniques that applied NOMA in multicell networks have been discussed in [5]. On the other hand, various NOMA-CoMP schemes have been analyzed in [7] and the study revealed that JT-NOMA-CoMP provides the maximum spectral efficiency gain. A coordinated superposition coding was proposed in [8] with Alamouti code employed at CoMP base stations. Besides, the authors in [9] presented a suboptimal scheduling strategy for NOMA-CoMP with linear complexity. An opportunistic NOMA-CoMP scheme was introduced in [10] to advance the capability of the system. However, the study assumed that the base stations and user devices are equipped with single antenna.

In this paper, we propose suboptimal resource allocation scheme for JT-NOMA-CoMP in downlink transmission with multiple antennas at base stations and user devices. In the first stage, available system bandwidth is allocated to the scheduled users. While in the later, transmission power is allocated to the respective scheduled users based on optimal power allocation policy. Then, we show the performance of the proposed scheme and compare to conventional JT-OMA-CoMP scheme. This paper is organized as follows. Section 2 describes the system model and Section 3 presents our proposed user clustering resource allocation algorithm. Section 4 shows the simulation criterion and the numerical results. Finally, the conclusion is drawn in Section 5.

\section{SYSTEM MODEL}

A JT-NOMA-CoMP considered in this study is illustrated in Figure 1. The CoMP system consists of $M$ base stations (BSs) having $N_{T}$ transmit antennas and $K$ mobile users (UEs) with $N_{R}$ receive antennas deployed in each cell. The UEs in each cell are divided into $L$ clusters and each cluster contains two users. Within a cluster, the user close to the BS with larger channel gain is defined as strong user, $u_{\text {near }}$, while the user far from the BS with smaller channel gain is known as weak user, $u_{f a r}$.

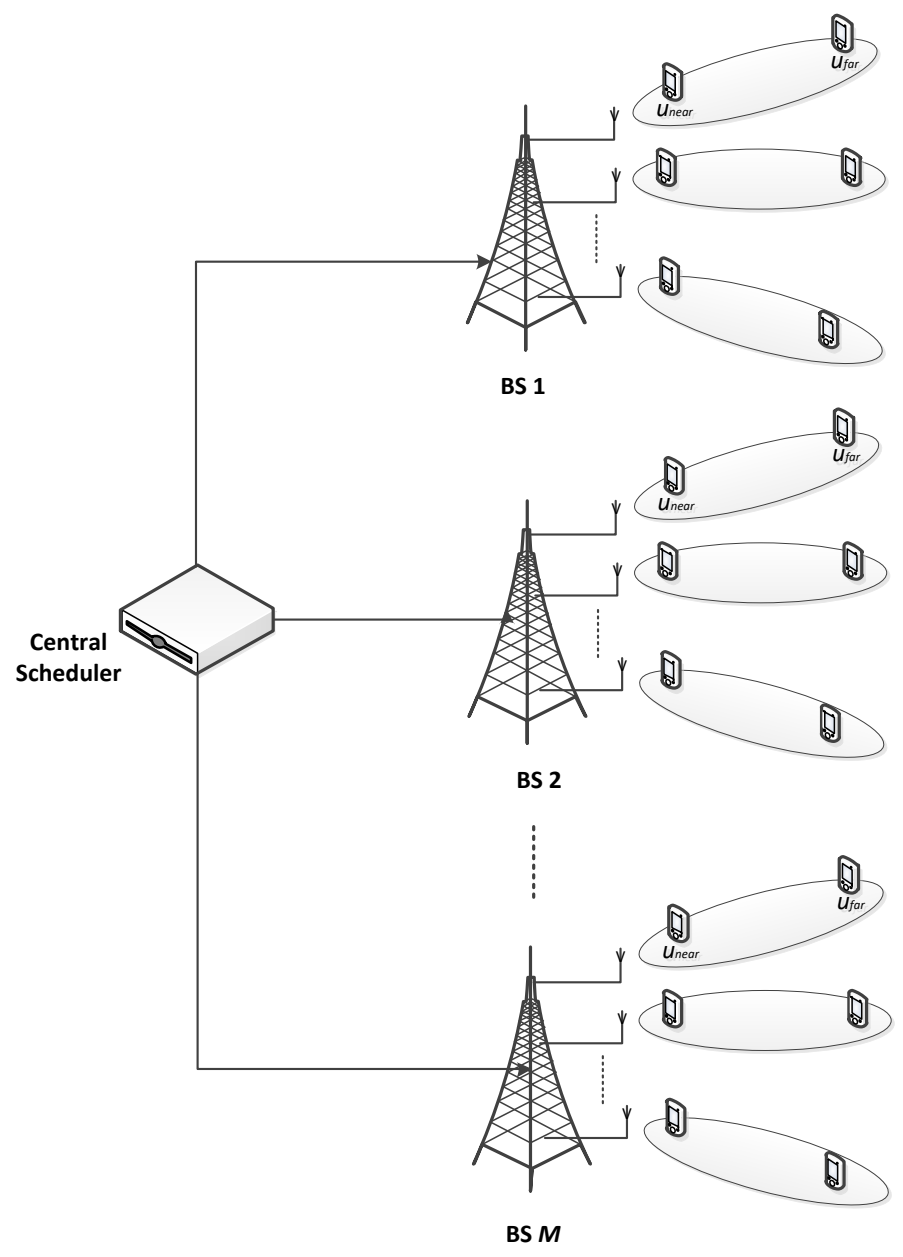

Figure 1. Downlink JP-NOMA-CoMP system 
The received signal at $u_{\text {near }}$ and $u_{\text {far }}$ can be expressed as;

$$
\begin{aligned}
& y_{\text {near }}=H_{\text {near }} x_{\text {near }}+H_{\text {near }} x_{\text {far }}+n_{\text {near }} \\
& y_{\text {far }}=H_{\text {far }} x_{\text {far }}+H_{\text {far }} x_{\text {near }}+n_{\text {far }}
\end{aligned}
$$

where $H_{\text {near }} \in \mathbb{C}^{N_{R} \times N_{T}}, H_{\text {far }} \in \mathbb{C}^{N_{R} \times N_{T}}$, denote the downlink channel of user $u_{\text {near }}$ and user $u_{f a r}$, respectively. $n_{\text {near }}, n_{\text {far }}$ are the normalized adaptive white Gaussian noise at $u_{\text {near }}, u_{\text {far }}$.

Based on NOMA principle, the SIC is realized at the receiver of $u_{\text {near }}$. In this scenario, $u_{f a r}$ will directly decode its own data, $x_{f a r}$ by treating the interference caused by $x_{\text {near }}$ as unknown interference. For $u_{\text {near }}$, it will first decode the data vector $x_{\text {far }}$ by treating the interference caused by $x_{\text {near }}$ as unknown interference and then cancel the part of received signal caused by $u_{f a r}$ from received signal and decode $x_{\text {near }}$ from the remain part of received signal.

Achievable data rates of near user, $R_{\text {near }}$ and far user, $R_{\text {far }}$ are bounded as follows:

$$
\begin{aligned}
& R_{\text {near }} \leq \log _{2}\left(1+\alpha P_{l}\left|H_{\text {near }}\right|^{2}\right) \\
& R_{\text {far }} \leq \log _{2}\left(1+\frac{(1-\alpha) P_{l}\left|H_{\text {far }}\right|^{2}}{\alpha P_{l}\left|H_{\text {far }}\right|^{2}}\right)
\end{aligned}
$$

where $P_{l}$ is transmit power allocated by the BS to antenna $n_{T}$, while $\alpha$ and $(1-\alpha)$ are the allocated power ratio of $u_{\text {near }}$ and $u_{\text {far }}$.

Hence, the sum rate in the $r$-th subchannel in cluster $l$ is given by

$$
R_{\text {sum }}^{r}=\log _{2}\left[\left(1+\alpha P_{l}\left|H_{\text {near }}\right|^{2}\right)\left(1+\frac{(1-\alpha) P_{l}\left|H_{\text {far }}\right|^{2}}{\alpha P_{l}\left|H_{\text {far }}\right|^{2}}\right)\right]
$$

All BSs in the system are connected to a central scheduler which responsible to manage available transmission resources in a coordinated way. The task is realized through information sharing over the backhaul network. In a JT-CoMP scheme with a multiple antenna BS and UE, multiple cells simultaneously transmit the same data to a set of scheduled CoMP users by using the same PRB [7]. Since the same data is transmitted by all CoMP cells, the reception performance of the CoMP users improves. In order to reduce the stringent requirements on backhaul network, selective combining (SC) is employed in this study, where only one BS is selected by the central scheduler to serve the user. The SC combiner chooses only the BS with the highest signal-to-noise ratio (SNR)

In this paper, we aim to realize a JP-NOMA-CoMP strategy so that the overall system sum rate can be maximized. In order to maximize the achievable data rates in (5), we will propose a user clustering followed by bandwidth and power allocation algorithm.

\section{PROPOSED APPROACH}

In this section, we provide detailed explanation on the proposed user clustering and resource allocation strategy. First, users in each cell are grouped based on the instantaneous channel gain. Then, available system bandwidth and power are allocated to the scheduled users.

\subsection{User clustering}

In NOMA, multiple users are allowed to use the same frequency bands simultaneously such that the signals of the users are superimposed with different power allocations. These multiplexed users are said to be in a user pair. Previous work from literature shown that high capacity gains can be attained if users with large channel gain difference are paired with each other [2, 11, 12].

Multiple antennas BS implies that more than one active transmission is possible at every time transmission interval (TTI). Optimal user clustering approach such as exhaustive search promises significant system capacity gain. However, due to the huge search space when multiuser multiple-input multiple output 
(MU-MIMO) environment is considered, the performance gain tends to be surpassed by the computational complexity.

To overcome this constraint, we propose a low-complexity user clustering algorithm based on the squared Frobenius norm of the MIMO channel, $\left\|H_{u_{k}}\right\|_{F}^{2} \cdot\left\|H_{u_{k}}\right\|_{F}^{2}$ is interpreted as a total power gain of a channel [13] that is;

$$
\left\|H_{u_{k}}\right\|_{F}^{2}=\sum_{i=1}^{N_{R}} \sum_{j=1}^{N_{T}}\left|h_{i, j}\right|^{2}=\sum_{i=1}^{N_{\min }} \lambda_{i}
$$

where $N_{\text {min }} \triangleq \min \left(N_{T}, N_{R}\right)$ and $\lambda_{i}$ are the eigenvalues of the Hermitian symmetric matrix, $H_{u_{k}} H_{u_{k}}^{H}$. In this paper, it is assumed that there are $K \times L, K \geq 2$ users in a cell and the BS serves $L$ clusters with $K$ users in each cluster. such that;

Next, the squared Frobenius norm values of users in each CoMP cell are ranked in descending order

$$
\left\|H_{u_{1}}\right\|_{F}^{2}>\left\|H_{u_{2}}\right\|_{F}^{2}>\left\|H_{u_{3}}\right\|_{F}^{2}>\cdots .>\left\|H_{u_{K}}\right\|_{F}^{2}
$$

The BS assigns the $k$-th user and $K M / 2+k$-th user into the $l$-th cluster, where $l=1,2,3, \ldots, K M / 2$.

\subsection{Bandwidth and power allocation}

There are $N_{R B}$ physical resource block (PRB), which represents the basic time-frequency resource unit for data transmission in LTE networks. A full buffer traffic model is considered. Throughout our study, the number of users that can be multiplexed over a physical resource block (PRB) is restricted to 2 .

Since each antenna beam of the BS is utilized by all the users of a cluster, the total BS transmit power, $P_{B S}$ is divided into the number of transmit antennas. such that :

$$
\sum_{l=1}^{N_{T}} P_{l} \leq P_{B S}
$$

where $P_{l}$ is transmit power allocated by the BS to antenna $n_{T}$. In this study, $P_{l}$ are equal for all antenna beams of a BS since the same cluster size (number of users per cluster) is adopted.

According to NOMA principle, at the BS side the intended message signals targeting to near user, $u_{\text {near }}$ and far user, $u_{f a r}$ are superimposed over PRB $r$, with transmit power $\alpha P_{l}$ and $(1-\alpha) P_{l}$ for $u_{\text {near }}$ and $u_{\text {far }}$, respectively.

Previous study shown that when the power difference between the $u_{\text {near }}$ and $u_{\text {far }}$ in a cluster becomes smaller, the SIC performance degrades [14-16]. Based on [16], if the difference between power allocation factors of both users is less than around 0.35, the drastic effects on the performance of both users start to occur; the SIC process at $u_{\text {near }}$ becomes imperfect and also the data recovery at $u_{f a r}$ is poorly affected. Hence, in our study, we assume that the power factor of $u_{\text {near }}$ and $u_{f a r}$ are kept constant at values 0.35 and 0.65 , respectively, as recommended in literature. This ensures that $u_{\text {near }}$ will be able to perform the SIC appropriately. Furthermore, the interference encountered by $u_{f a r}$ due to $u_{\text {near }}$ signal will also be well-managed.

\section{RESULTS AND DISCUSSION}

In the simulation system, the JP-NOMA-CoMP system with three cells is considered. Users are randomly distributed in the cell. The number of transmit antennas at each BS, $N_{T}=4$ and each user device is equipped with $N_{R}=2$ antennas. All the parameters and setting used in the simulation are tabulated in Table 1. Three different scenarios are considered in our simulation study;

Scenario 1: JP-OMA-CoMP with multiple transmit antenna at the BS employing water-filling (WF) power allocation [17]

Scenario 2: JP-NOMA-CoMP with single transmit antenna at the BS

Scenario 3: JP-NOMA-CoMP with multiple transmit antenna at the BS (Proposed approach) 
Table 1. Simulation parameters

\begin{tabular}{lc}
\hline \multicolumn{1}{c}{ Parameters } & Assumptions \\
\hline Cell Layout & Hexagonal 3 cell CoMP system \\
Channel Model & Rayleigh fading model \\
Radius of BS coverage & $500 \mathrm{~m}$ \\
BS transmit antenna & 4 \\
User terminat receive antenna & 2 \\
BS transmit power & $43 \mathrm{dBm}$ \\
Scheduling interval & $1 \mathrm{~ms}$ \\
PRB bandwidth & $180 \mathrm{kHz}$ \\
Path Loss Model & $128.1+37.6 \log (d$ in $\mathrm{km}) \mathrm{dB}$ \\
\hline
\end{tabular}

\subsection{Numerical results}

We carried out performance evaluation of the proposed approach (Scenario 3) and compared to JP-OMA-CoMP (Scenario 1) and JP-NOMA-CoMP (Scenario 2). Figure 2 depicts the achievable system sum rate versus total number of users deployed. It is clearly observed that the proposed approach (Scenario 3) provides the highest system achievable sum rate compared to Scenario 1 and Scenario 2. In Scenario 1, water-filling based (WF) power allocation algorithm is employed, where a channel with larger SNR is allocated more transmission power which consequently improves the system sum rate. Although the system in Scenario 1 acquires additional gains by utilizing WF power allocation and multiple antenna technique, its performance is inferior compared to Scenario 3. In Scenario 3, NOMA gives significant sum rate enhancement because multiple users are allowed to access the same PRB simultaneously. On the other hand, even both Scenario 2 and Scenario 3 adopt NOMA transmission, the system performance in Scenario 3 is superior compared to Scenario 2. The proposed strategy enhances system sum rate by taking advantage of multiuser diversity offered by multiple antenna technique. Furthermore, the central scheduler chooses a set of clusters that maximizes system sum rate.

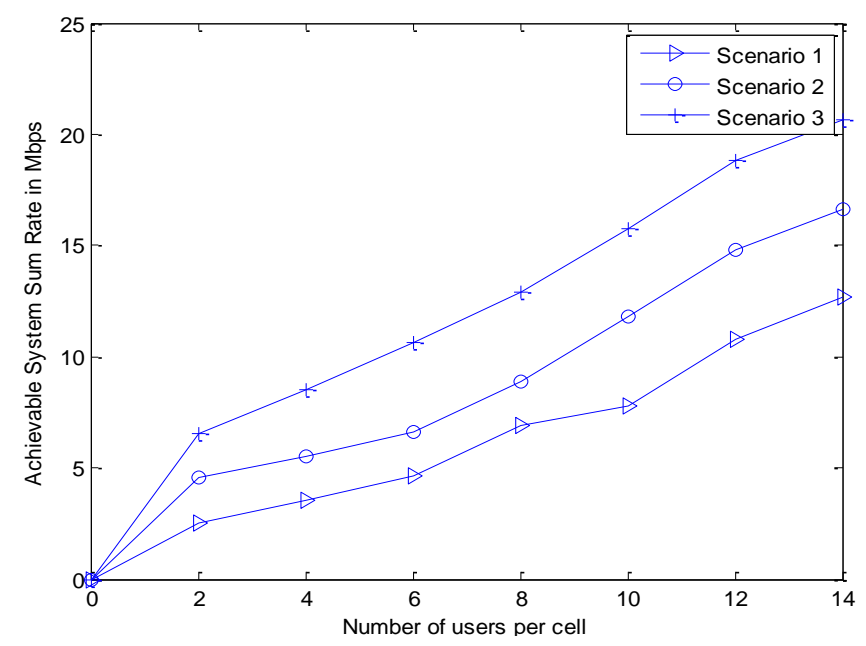

Figure 2. Performance comparison for Scenario 1, Scenario 2 and Scenario 3 in terms of achievable system sum rate

\section{CONCLUSIONS}

In this paper, a low-complexity user clustering and resource allocation scheme for dowblink JPNOMA-CoMP system have been investigated with the goal of maximizing the system sum rate. Numerical results show that the proposed approach yields a better performance than the other schemes in terms uf system sum rate. In future, optimal power allocation scheme which satify users' minimum data rate requirement will be considered

\section{ACKNOWLEDGEMENTS}

The research is funded by Research Management Centre (RMC), Universiti Tun Hussein Onn Malaysia (UTHM) under grant code H154 


\section{REFERENCES}

[1] G. P. Marsch, Patrick and Fettweis, Coordinated Multi-Point in Mobile Communications: from theory to practice. Cambridge University Press, 2011

[2] P. Sedtheetorn and T. Chulajata, "Accurate Spectral Efficiency Analysis for Non Orthogonal Multiple Access," vol. 5, no. 3, pp. 844-850, 2016.

[3] L. Dai, B. Wang, Y. Yuan, S. Han, C. L. I, and Z. Wang, "Non-orthogonal multiple access for 5G: Solutions, challenges, opportunities, and future research trends," IEEE Commun. Mag., vol. 53, no. 9, pp. 74-81, 2015.

[4] Z. Ding, Y. Liu, J. Choi, Q. Sun, M. Elkashlan, C.-L. I, and H. V. Poor, "Application of Non-orthogonal Multiple Access in LTE and 5G Networks," pp. 1-13, 2015.

[5] W. Shin, M. Vaezi, B. Lee, D. J. Love, J. Lee, and H. V. Poor, "Non-orthogonal multiple access in multi-cell networks: Theory, performance, and practical challenges," IEEE Commun. Mag., vol. 55, no. 10, pp. 176-183, 2017.

[6] Y. Wang, B. Ren, S. Sun, S. Kang, and X. Yue, “Analysis of Non-Orthogonal Multiple Access for 5G," no. 10, pp. 52-66.

[7] M. S. Ali, E. Hossain, and D. I. Kim, "Coordinated Multipoint Transmission in Downlink Multi-Cell NOMA Systems: Models and Spectral Efficiency Performance," IEEE Wirel. Commun., vol. 25, no. 2, pp. 24-31, 2018.

[8] J. Choi, "Non-orthogonal multiple access in downlink coordinated two-point systems," IEEE Commun. Lett., vol. 18 , no. 2, pp. 313-316, 2014.

[9] A. Beylerian, "Coordinated Non-Orthogonal Mulitple Access," 2016.

[10] Y. Tian, A. R. Nix, and M. Beach, "On the Performance of Opportunistic NOMA in Downlink CoMP Networks," IEEE Commun. Lett., vol. 20, no. 5, pp. 998-1001, 2016.

[11] J. He, Z. Tang, and Z. Che, "Fast and efficient user pairing and power allocation algorithm for non-orthogonal multiple access in cellular networks," Electron. Lett., vol. 52, no. 25, pp. 2065-2067, 2016.

[12] J. Mei, L. Yao, H. Long, and K. Zheng, "Joint user pairing and power allocation for downlink non-orthogonal multiple access systems," 2016 IEEE Int. Conf. Commun. ICC 2016, pp. 0-5, 2016.

[13] G. K. Yong, S. C., Jaekwon, K., Won, Y. Y. and Chung, MIMO-OFDM Wireless Communications with MATLAB. 2010.

[14] C. Yan, A. Harada, A. Benjebbour, Y. Lan, A. Li, and H. Jiang, "Receiver design for downlink non-orthogonal multiple access (NOMA)," IEEE Veh. Technol. Conf., vol. 2015, 2015.

[15] K. Saito, A. Benjebbour, A. Harada, Y. Kishiyama, and T. Nakamura, "Link-level performance of downlink NOMA with SIC receiver considering error vector magnitude," IEEE Veh. Technol. Conf., vol. 2015, pp. 6-10, 2015.

[16] K. Saito, A. Benjebbour, Y. Kishiyama, Y. Okumura, and T. Nakamura, "Performance and design of SIC receiver for downlink NOMA with open-loop SU-MIMO," 2015 IEEE Int. Conf. Commun. Work. ICCW 2015, pp. 1161-1165, 2015.

[17] N. Katiran, N. Fisal, S. K. S. Yusof, A. S. Abdul Ghaffar, S. M. Mohamad Maharum, and F. Asraf Saparudin, "Joint Power Allocation Strategy in CoMP ( JP ) Transmission," in IEEE Symposium on Wireless Technology and Applications, 2012.

\section{BIOGRAPHIES OF AUTHORS}

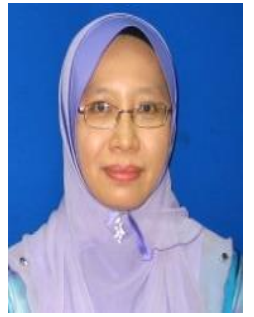

Norshidah Katiran received her Ph.D in Electrical Engineering from University Teknologi Malaysia, in 2015, her M.Eng. degree in Communication and Computer Engineering from University Kebangsaan Malaysia, in 2004, and her B.Eng. degree in Electrical Engineering (Telecommunications) from University Teknologi Malaysia, in 2001. Currently, she is a Senior Lecturer in the Department of Communication Engineering at University Tun Hussein Onn Malaysia. Her major research interests include optimization of resource allocation in cooperative networks and multiple input multiple output (MIMO) transmissions.

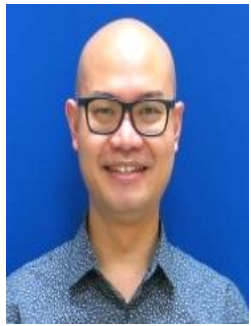

Shaharil Mohd Shah obtained his B. Eng in Microwave and Communication from Multimedia University (MMU) in 2002. He received his M.Sc in Microwave Engineering and Wireless Subsystems Design from the University of Surrey, UK in 2004 and pursuing his Ph.D in Communication Engineering from the University of Birmingham, UK before graduating in 2016. $\mathrm{He}$ is currently a senior lecturer in the Department of Communication Engineering, Faculty of Electrical and Electronic Engineering, Universiti Tun Hussein Onn Malaysia (UTHM). His area of research includes, but not limited to, design of microwave devices, active antennas measurement and nonlinear characterisation of active devices. 

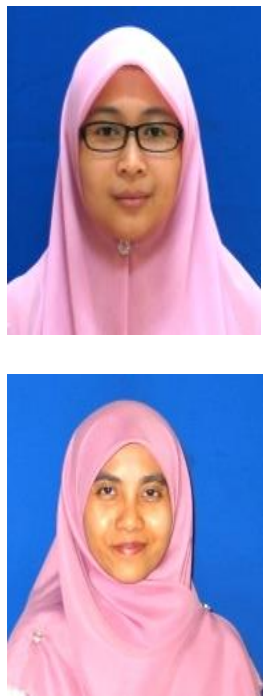

Aimi Syamimi Ab Ghafar received her B.Sc. in Electrical Engineering (Telecommunication) (2010) and PhD (2015) from Universiti Teknologi Malaysia. Currently, she serves as a lecturer at University Tun Hussein Onn Malaysia. Her current research interests are Next-Generation Mobile Technology, LTE-Advanced, cooperative relay communications, and resource allocation in wireless communication networks.

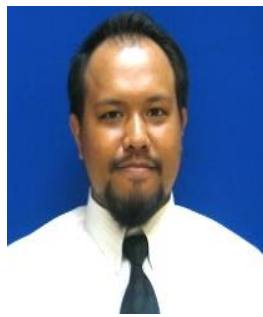

Faiz Asraf Saparudin received his Bachelor B.Sc. in Electrical Engineering (Telecommunication) with First Class Honours and Doctorate Degree from University Teknologi Malaysia, in 2010 and 2015, respectively. In 2010, he received WAMY Academic Excellence Award. He is currently working as a lecturer in University Tun Hussein Onn Malaysia. His current research interests include radio resource management, distributed algorithms, nature-inspired techniques, multiagent system and game theoretic approach for next-generation mobile network. 